\title{
MTDC SYSTEMS FOR FREQUENCY SUPPORT BASE ON DC VOLTAGE MANIPULATION
}

\author{
L. Xu ${ }^{1}$, J. Rafferty ${ }^{2}, Y$. Wang $^{3}$, and G. Xu ${ }^{3}$ \\ ${ }^{1}$ University of Strathclyde, Glasgow, G1 1XW, UK. Email: lie.xu@strath.ac.uk \\ ${ }^{2}$ ESBI, Cork, Ireland. Email: jrefferty@esbi.ie \\ ${ }^{3}$ North China Electric Power University, Baoding,China.Email: vi.wang@ncepu.edu.cn, guoyi.xu@ncepu.edu.cn
}

\section{Keywords: Droop control; MTDC; frequency support}

\begin{abstract}
This paper presents control strategies for multi-terminal HVDC systems to provide primary frequency support to connected AC networks via coordinated DC voltage manipulation. Inertia response from DC connected large offshore wind farms can also be incorporated without telecommunication based on the detection of DC voltage derivation. Simulation studies based on a 3-terminal HVDC system connecting one large wind farm and two separate AC networks validate the operation of the system during frequency events.
\end{abstract}

\section{Introduction}

Proposed large multi-terminal HVDC (MTDC) systems, incorporating a significant number of interconnected wind farms could result in the overall inertia of the system being substantially reduced making the system frequency more susceptible to changes in load [4]. In order for the MTDC systems to provide primary frequency response management strategies for any proposed DC grid will need to take into account the necessity for the system frequency to be maintained within designated levels. Additionally, even with utilization of wind turbine inertia response, allocation of the additional available power from inertia response is an issue for MTDC systems due to the increased number of converters involved and the decoupling of the $\mathrm{AC}$ and $\mathrm{DC}$ grids. Therefore effective DC grid management strategies will be required to optimize the full network support benefits available.

The ability of a MTDC system to provide frequency support has been investigated, however its capacity to do so is limited in these studies to the amount of available active power on the DC system, and/or the additional power generated from wind turbine inertia response $[1,6,10,11]$. Primary frequency support in MTDC systems has been proposed by the means of $\mathrm{DC}$ voltage and AC frequency droop control [10]. However, the work considers only minor frequency events on the system and results in a lower baseline common DC voltage even under normal conditions, in other words, no load imbalance. It also does not consider the utilization of wind turbine inertia for the provision of frequency support, nor does it consider the $\mathrm{AC}$ interconnection of the $\mathrm{DC}$ converter grid points, which could potentially exist within the proposed Supergrid, due to the amalgamation of the existing AC networks at the receiving end via AC (or DC) interconnectors between neighbouring $\mathrm{AC}$ grid points. This raises its own problems in terms of detection of frequency imbalances and subsequent support capabilities of HVDC converters due to the averaging of the frequency over a few seconds within all parts of a synchronous AC system [9]. Work has been carried out on the effect of the loss of a converter on the frequency deviation in a MTDC system, proposing a method of sharing the power imbalance between the remaining terminals to offset the frequency event [6]. However the model does not consider any "load priority" at the receiving end converters and the subsequent grid management requirements of ensure that sufficient active power is available to offset the load imbalances.

Another issue yet to be considered is that of detection of frequency events from a wind turbine perspective, a process which currently has to be carried out by long distance telecommunication due to the decoupled receiving end $\mathrm{AC}$ grid and wind farm AC system by the MTDC grid. This is not an ideal solution due to the possible loss of connection or lag time involved due to the substantial distances involved with offshore wind turbine HVDC interconnection.

The objective of this paper is to further access the capabilities of VSCs to provide frequency support to interconnected AC systems within MTDC systems during periods of under/over frequency. It proposes the redistribution of available active power in the MTDC system to offset the effect of load imbalances on interconnected AC networks utilizing the implementation of DC grid management strategies. It also proposes a telecommunication-less method of detection of frequency imbalances by HVDC interconnected variable speed wind turbines.

\section{Proposed system for case study}

The system proposed is a three-terminal VSC based MTDC system interconnecting two grid side receiving end VSCs and a single sending end VSC connected to an offshore wind farm (VSC1) as schematically shown in Figure 1. This portion of the system is adopted from a two-terminal model as proposed in [5]. The proposed DC gird has a nominal DC voltage of 


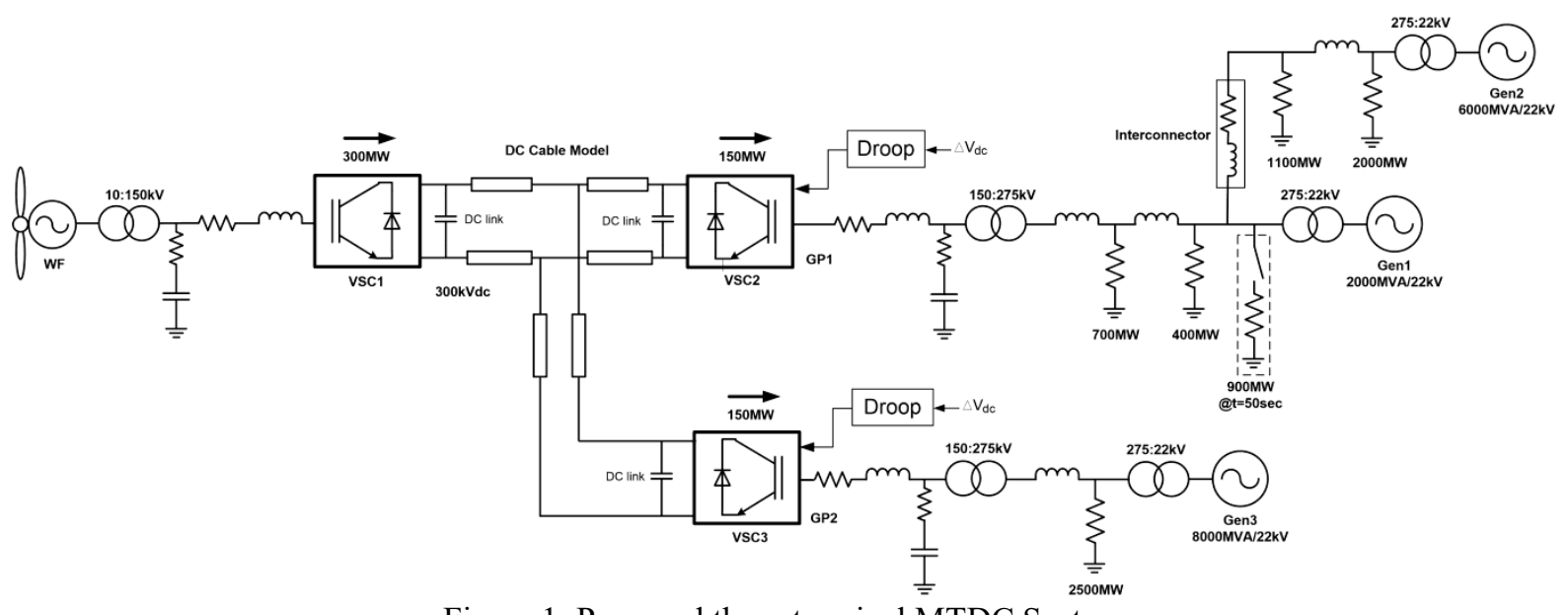

Figure 1: Proposed three-terminal MTDC System

$\pm 300 \mathrm{kV}$ interconnected with the two onshore $275 \mathrm{kV} \mathrm{AC}$ networks. The AC system at VSC2 (GP1) here is a single AC grid with two synchronous generators of 2000MW (Gen1) and 6000MW (Gen2) respectively, supplying a load of $4000 \mathrm{MW}$. The two generators are interconnected by a single $\mathrm{AC}$ interconnector, also operating at $275 \mathrm{kV} \mathrm{AC}$ with a maximum capacity of $600 \mathrm{MW}$. The AC system at the remaining receiving end $\mathrm{VSC} 3$, is modelled as a single synchronous generator of $8000 \mathrm{MW}$ (Gen3) and supplies a load of $5000 \mathrm{MW}$ under normal conditions. For the purposes of this study a $500 \mathrm{MW}$ offshore wind farm (WF) is connected to converter VSC1 and the wind farm is modelled as a lumped PMSG connected to VSC1 at $150 \mathrm{kV}$ offshore AC line.

\section{MTDC system for frequency support}

The ability of variable speed wind turbines to provide temporary additional active power from deceleration of the turbines rotor, thanks to the large stored turbine blade inertia for the provision of frequency support has been well documented [1]. Thus no more description is given here.

\subsection{Criteria for of Potential Frequency Support Methods}

As previously discussed, wind turbine inertia response can provide a sizable level of additional power to offset frequency events within the AC system in which large offshore wind farms are considered [2, 3, 8]. However, this is really only a temporary solution, since the issue of the reduction of the mechanical power turbine produces a shortfall in power that will need to be recouped eventually by the turbine as the rotor needs to accelerate back to its optimum speed. Therefore, the frequency support capacities from the wind turbine inertia are limited for two reasons:

1. Limited reduction in speed, therefore limited power extractable due to possibility of turbine stalling.

2. Reduction in mechanical kinetic power reduces overall power output of the turbine, as the turbine needs to accelerate back to normal speed.
Given these limitations, it would be advantageous if the system was capable of offsetting small scale frequency events, without the need for additional power from the turbine inertia. Further to this, in the event that inertia response is used, when multi-terminal systems with isolated AC networks are considered, it is necessary for the system to allocate the extracted additional power to the correct converter terminal to ensure that AC system experiencing the load imbalance is alleviated. Also, care must be taken to ensure that the proposed frequency support method does not compromise the stable operation of a terminal not experiencing a load imbalance. This could be caused by redistributing too much active power in an attempt to offset the load imbalance at the offending terminal, and will be dependent on the power requirements of the unoffending terminal. Different terminals in a MTDC grid can also be prioritized to allow the allocation of power dependant on the demand at the receiving ends while ensuring that security of supply is maintained.

Another issue that arises from the use of wind turbine inertia in MTDC systems is how the turbine detects when and at what terminal the imbalance has occurred, since the turbines are decoupled from the onshore AC grid. Communication between the receiving end terminal frequency measurement devices and the sending end converter is a possibility though with additional cost and reduced system reliability. This also applies to the connection of different power networks via MTDC systems.

\subsection{Frequency Support utilising DC Grid Management}

By utilisation of DC grid management strategies for the control of DC active power within the system, relocation of active power via DC converter control can be used to redistribute power away from low priority receiving end to offset the power imbalances, and hence frequency imbalances, at offending terminals. This redistribution of active power would result in the sharing of large frequency dips between multiple grid points thus resulting in smaller, more manageable frequency dips at each terminal, thus ensuring that the total frequency of the system remains within acceptable levels as outlined by the system operator. 
As previously discussed, it would be advantageous to keep telecommunication between DC terminals to a minimum, to minimise the response time of the system during frequency support and to eliminate any possible connection issues that can arise from long distance telecommunications. Therefore, in this section it is proposed that the provision of frequency support is to be based on locally measurements and system control.

For DC voltage droop control the DC current $\left(I_{d c}\right)$ is determined by the DC voltage error $\left(\Delta V_{d c}\right)$ as [7]

$$
\begin{aligned}
& \Delta V_{d c}=V_{d c}-V_{r e f} \\
& I_{d c}=k \cdot \Delta V_{d c}
\end{aligned}
$$

where $V_{\text {ref }}$ and $V_{d c}$ are the reference and measured DC voltages respectively, and $k$ is the droop constant.

The active power output at a converter terminal is given as

$$
P=V_{d c} \cdot I_{d c}
$$

Therefore, via manipulation of the set voltage reference of the droop controller we can manipulate the perceived voltage change by the controller

$$
V_{r e f} \downarrow \Rightarrow \Delta V_{d c} \uparrow \Rightarrow I_{d c} \uparrow \Rightarrow P \uparrow
$$

With regards to the method of manipulating the $V_{\text {ref }}$ value, there are two options available. The first is to increase/decrease the value of $V_{\text {ref }}$ dependent on whether the converter is detecting a frequency peak/dip at its respective AC grid point. In this example, the droop integral $k$ value will remain the same, independent of the DC voltage reference. The VI characteristic for a system utilizing this control method is as shown in Figure 2, where, $k_{\text {nom }}, k_{\text {dip }}, k_{\text {peak }}$ and $V_{\text {nom }}, V_{\text {dip }}, V_{\text {peak }}$ are the droop constants and reference voltages under normal, frequency dip and frequency surplus conditions, respectively.

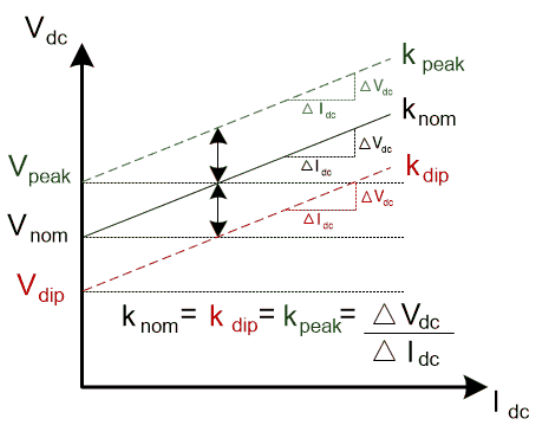

Figure 2: Frequency support option 1: normal and load imbalance DC voltage droop characteristics

Alternatively, manipulation of $\Delta V_{d c}$ can also be achieved by manipulation of the droop integral $k$ value directly. In this control option

$$
k_{\text {dip }} \neq k_{\text {nom }} \neq k_{\text {peak }}
$$

The VI characteristics are shown in Figure 3. Although this could potentially achieve similar frequency support capabilities manipulation of the integral $k$ values in real time could present its own challenges, particularly as the actual power sharing between the two converters would be dependent on not only the $k$ value, but also the cable resistance.

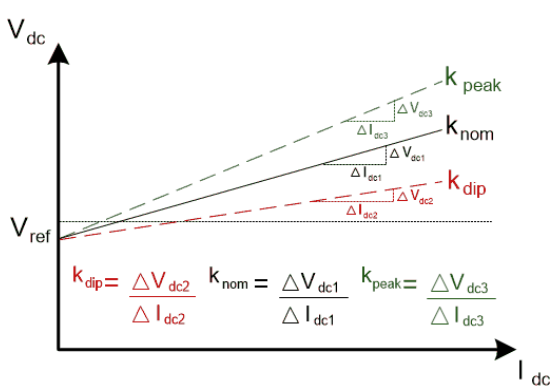

Figure 3: Frequency support option 2: normal and load imbalance DC voltage droop characteristics

Therefore, the proposed frequency support (FS) algorithm utilizes Option 1 and is shown in Figure 4. It should be noted that $k_{f}$ is the frequency event constant, and is provided to allow a certain degree of flexibility within the system. Differing $k_{f}$ values affect the system behavior though there are not discussed here due to space limitations. In Figure 4, Freq $_{V S C l}$ and Freq $_{\text {ref }}$ are the measured and reference frequencies at the VSC connection point respectively, $\Delta$ freq, $f_{\text {over limit }}$ and $f_{\text {under limit }}$ are the change in frequency, and over and under frequency threshold limits respectively.

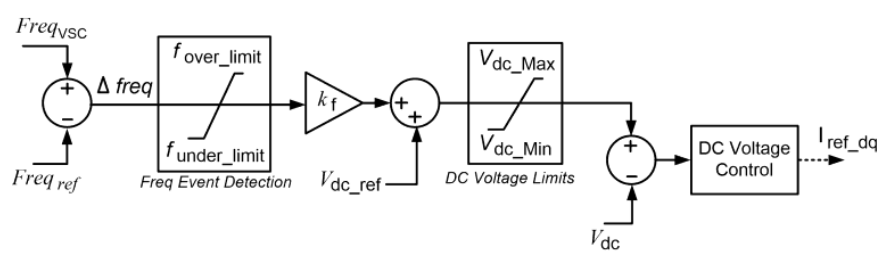

Figure 4: Frequency support algorithm

Due to the flexible nature of the DC droop control at VSC2 and VSC3 automatic readjustment of the unaffected converter when power is redistributed to an offending terminal is achieved, without the need for additional intelligent control, thus reducing lag time to a minimum. If DC voltage control utilizing a PI controller is more suitable to meeting the control requirements of the system it is proposed that the system operate in two separate modes, namely: Normal (PI Control) and Imbalance operation, where the converter terminal would switch to DC droop control when a load imbalance is detected. The effect of this control algorithm when a PI controller is utilized at a receiving end VSC is not given here again due to space limitation.

A graphical depiction of the proposed power redistribution method for frequency support is shown in Figure 5, where $P_{W F}$ and $P_{I R}$ are the normal power output and additional power output from wind turbine inertia response from VSC1 respectively. 


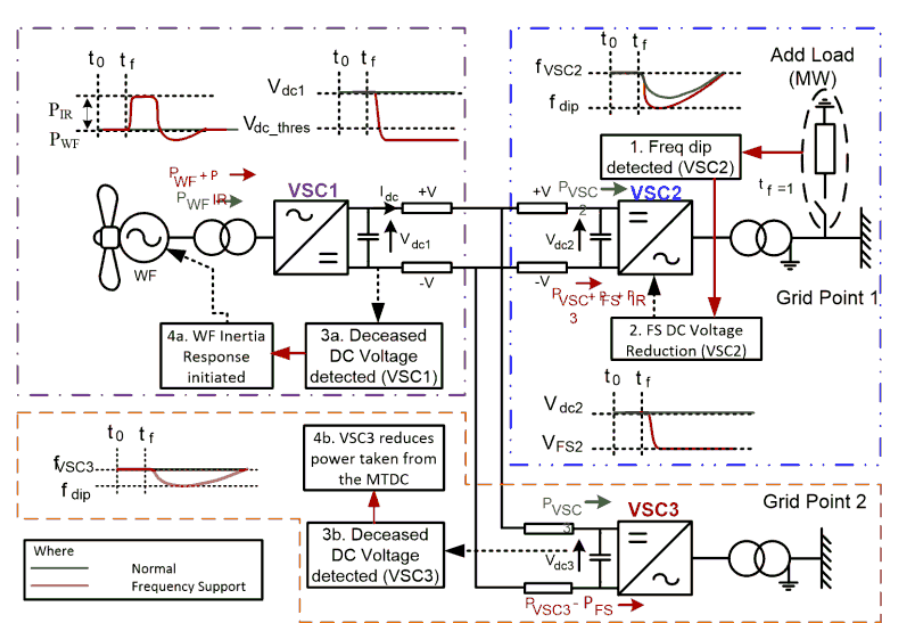

Figure 5: Frequency Dip/Inertia Response Detection based on Shared DC Voltage in MTDC System

\subsection{Detection of Frequency Events at Isolated Wind Farms within MTDC}

Another characteristic of this frequency support via manipulation of the DC voltage method is that changes in the DC voltage at a single terminal are reflected at all terminals within the MTDC system. It could potentially provide a means by which a decoupled offshore wind farm (or a DC connected independent AC network) can be informed of the occurrence of a frequency event on the onshore/other $\mathrm{AC}$ system and hence, provide additional power via either inertia response or power redistribution.

Taking wind farm as an example, the drop in DC voltage at the wind farm terminal can be used to detect that the receiving AC system is suffering a load imbalance, and it can, therefore, supply additional active power to the DC system via inertia response. This has the advantage of being automatic and telecommunication-less, though this reduction in the DC voltage has to be limited, say within $10 \%$ of nominal DC voltage such that normal operation of the system is not affected. By using the proposed controller, the drop of $\mathrm{AC}$ system frequency could be reflected by the drop of the DC link voltage. As the DC link voltage variation is almost the same along the DC transmission line, thus DC link voltage measured at the wind farm side converter can be used as an indicator for the AC system's requirement for the frequency support from the offshore wind farm. The DC voltage could also be used to modulate the frequency of the wind farm collection system by add an additional controller to the wind farm side VSC. Then the frequency link between the main $\mathrm{AC}$ network and the offshore wind farm collection network is re-established, and the wind turbines can be controlled to emulate the inertial response to provide frequency support to the main network. Another option is to directly use the DC link voltage to activate the inertial response of the wind turbines. For this strategy, a voltage relay as shown in Figure 6 can be used. Once the DC voltage drops below $\Delta V_{d c n}$ the inertial response is activated by the flag signal which is communicated from the wind farm side
VSC and the wind turbines. Wind turbine inertial response can be implemented by the methods proposed in [4].

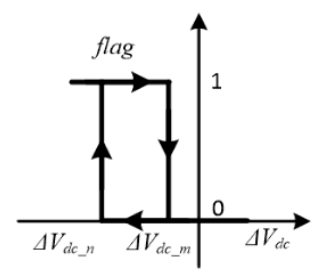

Figure 6: Voltage relay.

\section{Case studies}

Frequency event case studies where two isolated AC systems connected at each grid side receiving end VSCs as shown in Figure 1 are carried out. In this case study, a significant load imbalance is simulated by an increase in the demand at GP1 (VSC2) of $550 \mathrm{MW}$ at time $\mathrm{t}=50 \mathrm{~s}$, resulting in a frequency nadir of approximately $0.3 \%(50 \mathrm{~Hz}$ to $49.85 \mathrm{~Hz})$, as can be observed in Fig. 7 (a) (the curve with No FS). It is important to note that in this example the total available power on the DC grid is insufficient to offset the load imbalance at VSC2.

\subsection{Frequency Support Algorithm Utilised at VSC2}

For this example it is assumed that there is no inertia response available from the wind farm. When frequency support is utilised, the dip in the $\mathrm{AC}$ frequency below the under frequency threshold of the GP1 system, detected by VSC2 causes the FS algorithm to activate, resulting in an automatic decrease in the DC voltage reference at VSC2 in relation to the size of the frequency dip, as can be observed in Figure 7 (e). This informs the DC system that additional power is required at $\mathrm{VSC} 2$, therefore diverting power away from VSC3 (Figure $7(d)$ ) results in an increase the active power supplied to VSC2 as seen in Figure 7 (c), in an attempt to offset the frequency dip. In this example, the frequency dip presents an issue in that even with the full available DC power supplied to VSC2 and the power demand still exceeds the supply.

In this instance DC grid management can be utilised to determine if another converter within the multi-terminal system is operating at enough of a power surplus to alleviate the power deficit at VSC2. Here, we assume that the GP2 system (VSC3) has a power surplus of $350 \mathrm{MW}$ that it can supply to the DC grid for the provision of frequency support. Due to the terminal classification previously outlined, VSC3 at GP2 is designated as "low priority load terminal" and it can therefore switch from an inverter (receiving) to a rectifier (sending) converter thus supplying additional active power to the DC system, as can be observed in Figure 7 (d). The exact capacity of a VSC operating in this fashion, in other words, the level of additional active power it can supply to the DC system would need be limited by the system operator to ensure system stability was maintained on its corresponding AC system. This redistribution of power at VSC2 and VSC3 causes a decrease in the frequency at VSC3 (although still within acceptable levels) and an offsetting of the frequency 
dip at VSC2, thus the magnitude of the frequency event is again shared between both $\mathrm{AC}$ networks with each remaining within acceptable levels for operational frequency as can be observed in Figures 7 (a) and (b).

In should be noted that, although the common DC voltage experiences a larger drop than the previous example, due to the necessity to redistribute more power in this case, the system still operates within grid code specifications as can be observed in Figures 7 (e) and (f). Naturally, there is a lower limit to which the DC voltage is allowed to drop before the system is compromised, thus the amount of active power redistributed has a finite limit. Again, these limits should be outlined and enforced by the DC grid operator to ensure safe and effective operation of the DC system and prevent the false detection of a DC cable fault under these circumstances.

\subsection{Frequency Support and Inertia Response Utilised (FS VSC2/IR (100MW))}

It should be noted that due to utilisation of the FS algorithm the triggering of the wind turbine inertia response is based on the shared DC voltage drop as outlined in Section 3.2. With both the frequency support algorithm and the inertia response utilised, the small amount of active power released from the turbines kinetic energy makes minimum contribution to the offsetting of the frequency imbalance but does help offset the initial frequency nadir at VSC2 when compared to the system utilising solely the FS algorithm, as can be observed in Figure7 (a) (FS VSC2/IR vs. FS@VSC2). Also, this additional power does help to offset the frequency nadir of VSC3 which arises from the sharing of the load imbalance between the receiving end VSCs by compensating for some of the power imbalance at VSC2, thus the amount of power supplied to the DC system by VSC3 for frequency support is initially reduced as can be observed in Figure 7 (f). However, again this additional power must be recouped by accelerating the turbine back to nominal speed thus leading to a decrease in the active power available on the DC system and hence the FS algorithm reduces the DC voltage at VSC2 once the inertia response has finished, to increase the power supplied to VSC2 to offset the frequency dip as can be in Figures 7 (c) - (d) at $\mathrm{t}=60 \mathrm{~s}$. It should also be observed that due to the reacceleration of the turbine post inertia response a second frequency dip occurs at VSC 3 at $\mathrm{t}=62 \mathrm{~s}$, however this is less than the initial frequency nadir, since the system frequency is recovering.

\section{Conclusions}

This paper investigates the capacity of multi-terminal HVDC systems to offset frequency events on the connected $\mathrm{AC}$ system via the regulation of the DC active power at each terminal. A three-terminal MTDC was modelled and used as a case study. Extraction of additional electric power from wind turbine inertia is a viable means of providing short term frequency support to the onshore $\mathrm{AC}$ network by reducing the power imbalance. It was shown that via manipulation of the DC voltage, a long distance telecommunication-less method
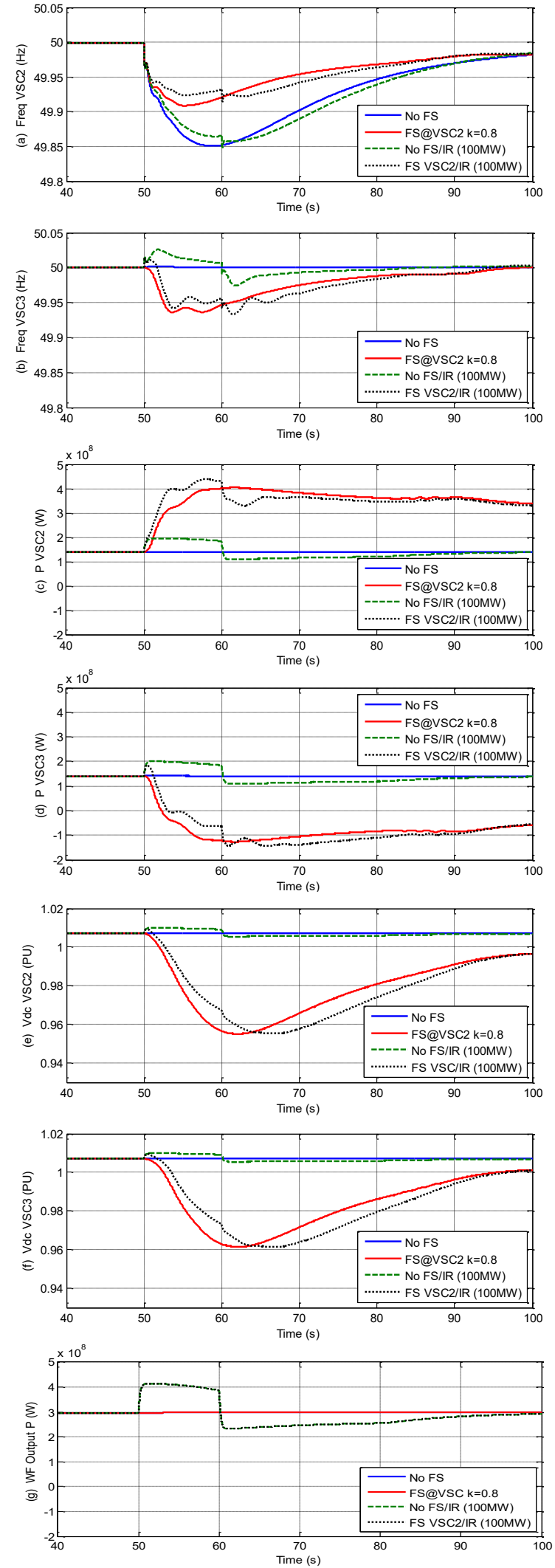

Figure 7: Simulation results during single load imbalance L1 at GP1 (550MW) applied at $50 \mathrm{~s}$ (isolated networks), where FS denotes a system utilising frequency support control algorithm, and IR denotes that the system is utilising inertia response from the wind farm. 
of frequency event detection for a DC grid connected wind farm was achievable.

For larger frequency events caused by larger load imbalances between multi-terminal systems it was proposed that a redistribution of the available active power to the offending $\mathrm{AC}$ system via the interconnected DC network could be utilised through manipulating the DC voltage within the system. This was achieved through a DC grid management strategy that allowed a "low priority" DC terminal within the system to provide active power to the DC grid dependant on necessity and availability. It was shown that utilization of a DC voltage droop controller at both sending end terminals allows the converter not experiencing a frequency dip to automatically readjust to the increased power demand of the converter experiencing the frequency dip, thus stable system operation is maintained.

\section{Acknowledgements}

This work was supported in part by the State Key Laboratory of Alternate Electrical Power System with Renewable Energy, China.

\section{References}

[1] A. B. T. Attya, T. Hartkopf, "Control and Quantification of Kinetic Energy released by Wind Farms during Power System Frequency Drops," IET Renewable Power Generation, Vol. 7, Iss. 3, pp. 210-224, May 2013.

[2] G. C. Tarnowski, P. C. Kjær, P. E. Sørensen and J. Østergaard, "Variable speed wind turbines capability for temporary over production," IEEE PES General Meeting, July. 2010.

[3] G. Ramtharan, J. B. Ekanayake, N. Jenkins, "Frequency Support from Doubly Fed Induction Generator Wind
Turbines," IET Renew. Power Gen., Vol.1, pp.2-9, 2007.

[4] G. Xu, L. Xu, D. J. Morrow, "System Frequency Support using Wind Turbine Kinetic Energy and Energy Storage," IET Renewable Power Generation Conference, Beijing, Sept. 2014.

[5] G. Xu, "Power Network Support using Wind Turbines with Embedded Energy Storage," PhD Thesis, Queen's University Belfast, March 2013.

[6] J. Ekanayake. N. Jenkins, "Comparison of the Response of Doubly Fed Fixed-Speed Induction Generator Wind Turbines to Changes in Network Frequency," IEEE Trans. on Energy Conversion, Vol. 19, No. 4, pp. $800-$ 802, Dec 2004.

[7] L. Xu, L. Yao, "DC Voltage Control and Power Dispatch of a Multi-Terminal HVDC System for Integrating Large Offshore Wind Farms" IET Renew. Power Gen., April 2011.

[8] N. R. Ullah, T. Thiringer and D. Karlsson, "Temporary primary frequency control support by variable speed wind turbines- potential and applications," IEEE Trans. Power Systems, Vol. 23, No. 2, pp. 601-612, May. 2008.

[9] T. M. Haileselassie, K. Uhlen, "Frequency Sensitivity Analysis of AC Grids Connected to MTDC Grid" IET conf. ACDC Power Tranmission., London, Oct. 2010.

[10] T. M. Haileselassie, R. Torres-Olguin, T. Vrana, K. Uhlen, T. Undeland, "Main Grid Frequency Support Strategy for VSC-HVDC Connected Wind Farms with Variable Speed Wind Turbines," IEEE Conc. PowerTech, Trondheim, June 2011.

[11] Y. Sun, Z. Zhang, G. Li, J. Lin, "Review on Frequency Control of Power Systems with Wind Penetration," IEEE conf. on Power System Technology (POWERCON), Hangzhou, Oct. 2010. 